\title{
Coded Modulation Assisted Iterative Parallel Interference Cancellation Aided CDMA
}

\author{
H.Wei, S.X.Ng and L.Hanzo ${ }^{1}$ \\ Dept. of ECS, University of Southampton, SO17 1BJ, UK. \\ Tel: +44-23-8059-3125, Fax: +44-23-8059-4508 \\ Email:1h ${ }^{1}$ @ecs.soton.ac.uk, http://www-mobile.ecs.soton.ac.uk
}

\begin{abstract}
In this contribution we investigate Trellis Coded Modulation (TCM) and Turbo Trellis Coded Modulation (TTCM) assisted iterative parallel interference cancellation aided CDMA schemes. The symbol-based Maximum a Posteriori (MAP) algorithm is invoked in both the TTCM and TCM decoder. In the context of the 8PSK TCM/TTCM CDMA schemes typically two iterations are necessary for approaching the best possible performance attained with the aid of an infinite number of iterations. By contrast, the number of iterations has to be increased to three or four in conjunction with 16QAM when the effective information throughput is increased from 2 to 3 bits per symbol.
\end{abstract}

\section{INTRODUCTION}

Following the philosophy of iterative turbo equalisation [1] in recent years iterative multiuser detection captured growing interest in the wireless communications community. In reference [2] an iterative decoding scheme designed for synchronous Code Division Multiple Access (CDMA) systems has been characterised. The algorithm proposed in [2] has a computational complexity proportional to the order of $O\left(2^{K v}\right)$, where $K$ is the number of users supported, and $v$ is the channel codec's memory length. This iterative multiuser detector exhibits near single-user performance. However, its excessive complexity renders its real-time implementations unrealistic, except when the number of users supported is low.

In references [3] and [4] several schemes have been proposed for reducing the implementational complexity imposed. In the set of these schemes, Interference Cancellation (IC) based iterative multiuser detection exhibits the lowest complexity, rendering the complexity proportional to the order of $O\left(K \cdot 2^{v}\right)$.

In reference [5] an IC based iterative Multiuser Detector (MUD) was developed, which exhibits a further reduced implementational complexity. The iterative re-

The financial support of the Mobile VCE, UK is gratefully acknowledged. ceiver advocated in this treatise obeys a similar structure to that proposed in [5], except for employing different channel coding and modulation schemes. In [3] and [4] the proposed iterative multiuser detectors invoked soft estimation of the real-valued BPSK modulated signal. In our approach, we propose a novel symbol probability based iterative multiuser detection assisted receiver for employment in a Trellis Coded Modulation (TCM) and Turbo Trellis Coded Modulation (TTCM) aided system. Symbol-based Maximum APosteriori (MAP) algorithms [6] are employed for both the TCM and TTCM decoder, which are capable of feeding back the symbol probabilities to the soft-symbol estimation and interference cancellation stages. More specifically, this algorithm relies on utilizing the probabilities of the channel-coded information symbols for symbol estimation, rather than on employing the individual APP of the coded TCM/TTCM bits, which was justified both experimentally as well as from a heuristic perspective in [7], when employing a Rake receiver. A turbo MUD was analysed in detail from a unified theoretical perspective in the excellent treatise of Boutros and Caire [8] under the assumption of using random spreading. ${ }^{1}$

This contribution is organized as follows. In Section 2 we briefly introduce the concept of the PIC scheme, while in Section 3 we describe the soft estimation of the coded symbols. In Section 4 we discuss the TCM/TTCM aided PIC based iterative detector in detail, while in Section 5 we characterise its achievable performance. Finally, in Section 6 we offer our conclusions.

\section{SOFT PARALLEL INTERFERENCE CANCELLATION}

Figure 1 shows the structure of a parallel interference cancellation scheme, where the soft estimates $\hat{b}_{k}, \quad k=$ $1, \ldots, K$ of the transmitted symbols, which are output by the channel decoder, are utilized for reconstructing estimates of the transmitted signal of all the $K$ users. Then, for each user, the reconstructed estimated signals of all the interfering users are subtracted from the composite multiuser signal and the resultant decontaminated signal

\footnotetext{
${ }^{1}$ The authors are grateful to the anonymous reviewer for his in-
} sightful remarks in this context. 
is processed by a Matched Filter (MF) or RAKE receiver, generating the soft output $\hat{\mathbf{y}}_{k}$. These modulated signal reconstruction, interference cancellation and re-estimation steps are repeated, until the affordable number of iterations is exhausted.

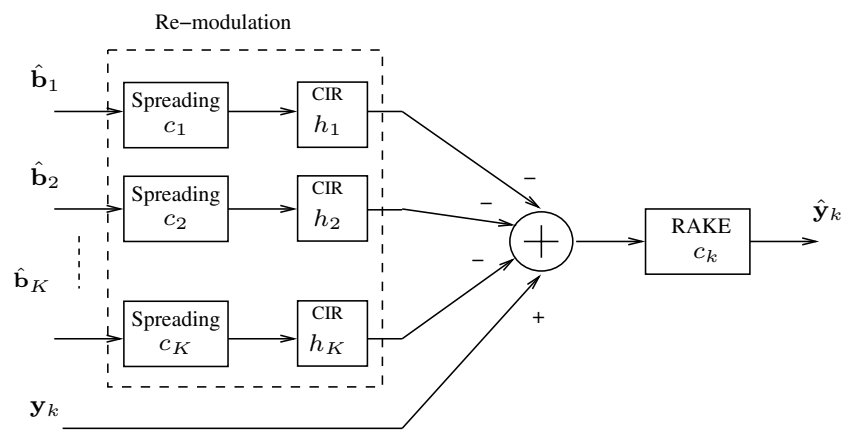

Figure 1: Schematic of a single PIC cancellation stage.

\section{ESTIMATION OF THE $M$-ARY CODED SYMBOL}

We employed rate- $m /(m+1) M=2^{m+1}$-ary TTCM and TCM schemes, where $m$ is the number of original uncoded information bits per symbol. Again, the symbol-based MAP [6] algorithm was invoked.The $k$ th user's TCM/TTCM decoder computes the soft symbol probabilities $\operatorname{Pr}\left\{b_{k}=\right.$ $\left.x_{i} \mid \hat{\mathbf{y}}_{k}\right\}, i=1, \ldots, M$ for each of the $K$ users, from the vector $\hat{\mathbf{y}}_{k}=\left[\hat{y}_{k, 1}, \ldots, \hat{y}_{k, t}, \ldots, \hat{y}_{k, L}\right]$. More specifically, the index $t, t=1, \ldots, T$ denotes the symbol index, $T$ is the decoding block length and $\hat{y}_{k, t}$ is the $k$ th user's estimated soft output generated by the soft PIC block at time instant $t$. Explicitly, the probability $\operatorname{Pr}\left\{b_{k}=x_{i} \mid \hat{\mathbf{y}}_{k}\right\}$ is the A Posteriori Probability (APP) of the $(m+1)$-bit TCM/TTCM symbol. Let us drop the user index $k$ and describe the TCM/TTCM decoder's operation in the context of a particular user. In the trellis decoding diagram, a branch leading from state $S_{t-1}=\grave{s}$ to state $S_{t}=s$ at time instant $t$ can be labelled by the original uncoded information symbol $a_{t}=u$ and the corresponding TCM/TTCM symbol $b_{t}=x_{i}, \quad i=1, \ldots, M$. Let us first denote the output of the soft MAI canceller $\hat{y}$ at time $t$ by:

$$
\eta_{t}(\grave{s}, s)=\hat{y}_{t} .
$$

The metric of the trellis branch leading from state $S_{t-1}=$ $\grave{s}$ to state $S_{t}=s$ can be written as:

$$
\gamma_{t}(\grave{s}, s)=\Pi_{t, u} \cdot \eta_{t}(\grave{s}, s),
$$

where the a priori probabilities $\Pi_{t, u}$ are either all equal to $1 / 2^{m}$ in the TCM scheme or are obtained from the other component decoder in the TTCM scheme. The above values are then used for recursively computing the forward recursion $\alpha_{t-1}(\grave{s})$ as:

$$
\alpha_{t}(s)=\sum_{\text {all } \grave{s}} \gamma_{t}(\grave{s}, s) \cdot \alpha_{t-1}(\grave{s}),
$$

and the backward recursion $\beta_{t}(s)$ as:

$$
\beta_{t-1}(\grave{s})=\sum_{\text {all } s} \beta_{t}(s) \cdot \gamma_{t}(\grave{s}, s) .
$$

The APP of the $(m+1)$-bit coded symbol at time $t$ can be obtained from:

$$
\operatorname{Pr}\left\{b_{t}=x_{i} \mid \hat{\mathbf{y}}\right\}(t)=\sum_{\substack{(\grave{s}, s) \Rightarrow \\ b_{t}=x_{i}}} \beta_{t}(s) \cdot \alpha_{t-1}(\grave{s}) \cdot \gamma_{t}(\grave{s}, s),
$$

where $(\grave{s}, s) \Rightarrow b_{t}=x_{i}$ indicates the specific set of transitions emerging from the previous state $S_{t-1}=\grave{s}$ to the present state $S_{t}=s$ that can be encountered, when the coded symbol is $b_{t}=x_{i}$.

The APPs of the coded symbols are then passed to the soft estimation block of Figure 2. Let us now drop the time index $t$ and reinstate the user index $k$. The estimated symbol of user $k$ at a particular time instant can be obtained from the soft estimation block of Figure 2 as:

$$
\hat{b}_{k}=\sum_{i=1}^{M} \operatorname{Pr}\left\{b_{k}=x_{i} \mid \hat{\mathbf{y}}_{k}\right\} \cdot x_{i},
$$

where $x_{i}$ is the complex-valued TCM/TTCM symbol. Finally, $\hat{b}_{k}$ is fed to the soft MAI cancellation unit of Figure 1 and the TCM/TTCM assisted iterative multiuser detection continues iteratively until the last iteration.

\section{ITERATIVE MULTIUSER DETECTION}

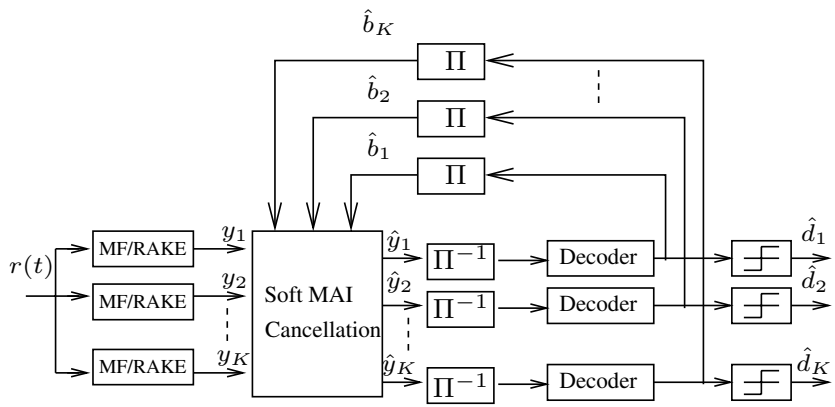

Figure 2: Schematic of the iterative PIC-aided detector.

Figure 2 shows the schematic of the PIC assisted iterative multiuser detector, where the Matched Filter's (MF) output is given by: $\mathbf{y}=\left[y_{1}, \ldots, y_{K}\right]^{T}$. The vector $\hat{\mathbf{b}}=\left[\hat{b}_{1}, \ldots ., \hat{b}_{K}\right]^{T}$ contains the estimated symbol of all the $K$ users, which includes the symbol probabilities 
generated by Equation 6. Once we obtained the soft symbol estimates, soft MAI cancellation is performed, yielding the decontaminated soft output signal vector $\hat{\mathbf{y}}=$ $\left[\hat{y}_{1}, \ldots, \hat{y}_{K}\right]^{T}$, where the blocks $\Pi$ and $\Pi^{-1}$ in Figure 2 denote the channel interleaver and deinterleaver, respectively.

\subsection{Synchronous System}

Let us now consider the special case, when the synchronous DS-CDMA system communicates over a non-dispersive Additive White Gaussian Noise (AWGN) channel. According to [9], we can express the received signal vector $\mathbf{y}$ at the output of the MF seen in Figure 1 as:

$$
\mathbf{y}=\mathbf{R A b}+\mathbf{n},
$$

where $\mathbf{R}$ is the normalized $K \times K$-dimensional cross-correlation matrix of the $K$ users' spreading codes. The element of the $i$ th row and $j$ th column of the matrix is denoted as $\rho_{i j}$, and we have $\rho_{i j}=\rho_{j i}$. Furthermore, the transmitted symbol vector $\mathbf{b}$ is expressed as: $\mathbf{b}=$ $\left[b_{1}, \ldots, b_{K}\right]^{T}$, and $\mathbf{n}=\left[n_{1}, \ldots, n_{K}\right]^{T}$ is the $(K \times 1)$-dimensiona noise sample vector at the output of the $\mathrm{MF}$, while

$\mathbf{A}=\operatorname{diag}\left\{A_{1}, \ldots, A_{K}\right\}$ is a diagonal matrix containing the energy-per-bit values for all the $K$ users.

Let us define $\hat{\mathbf{b}}_{k}$ as a vector, which equals $\hat{\mathbf{b}}$ except that its $k$ th element is zero. Then the vector $\hat{\mathbf{y}}_{k}$ containing the desired signal of user $k$ in its $k$ th position can be written as:

$$
\hat{\mathbf{y}}_{k}=\mathbf{R A}\left(\mathbf{b}-\hat{\mathbf{b}}_{k}\right)+\mathbf{n}, k=1, \ldots, K
$$

Explicitly, once a reliable soft estimate $\hat{\mathbf{b}}$ becomes available, the vector $\hat{\mathbf{y}}$ becomes free from MAI and hence it is contaminated only by the channel noise.

\subsection{Asynchronous System}

In an asynchronous system the symbols transmitted during the $i$ th signalling interval are also affected by all the asynchronous interfering signals transmitted during the intervals $(i+1)$ and $(i-1)$. Hence, according to [9], for an asynchronous DS-CDMA system communicating over a non-dispersive AWGN channel the received signal vector y can also be expressed as:

$$
\mathbf{y}[i]=\mathbf{R}^{T}[1] \mathbf{A b}[i+1]+\mathbf{R}[0] \mathbf{A} \mathbf{b}[i]+\mathbf{R}[1] \mathbf{A b}[i-1]+\mathbf{n}[i]
$$

where $i$ is the index of time instant and the zero-mean Gaussian channel noise process $\mathbf{n}[i]$ has the crosscorrelation matrix:

$$
E\left[\mathbf{n}[i] \mathbf{n}^{T}[j]\right]= \begin{cases}\sigma^{2} \mathbf{R}^{T}[1], & \text { if } j=i+1 \\ \sigma^{2} \mathbf{R}[0], & \text { if } j=i \\ \sigma^{2} \mathbf{R}[1], & \text { if } j=i-1 \\ \mathbf{0}, & \text { otherwise }\end{cases}
$$

while the matrix $\mathbf{R}[1]$ and $\mathbf{R}[0]$ are defined as:

$$
\begin{aligned}
& \mathbf{R}_{j k}[0]= \begin{cases}1, & \text { if } \mathrm{j}=\mathrm{k} ; \\
\rho_{j k}, & \text { if } j<k ; \\
\rho_{k j}, & \text { if } j>k,\end{cases} \\
& \mathbf{R}_{j k}[1]= \begin{cases}0, & \text { if } j \geq k ; \\
\rho_{k j}, & \text { if } j<k .\end{cases}
\end{aligned}
$$

Let us now define the vectors $\hat{\mathbf{b}}_{k}[i+1], \hat{\mathbf{b}}_{k}[i], \hat{\mathbf{b}}_{k}[i-1]$ as the soft estimates of the $k$ th user during the signalling instants $(i+1), i$, and $i-1$, which have had their $k$ th element set to zero. Therefore, after the soft MAI cancellation stage of Figure 2, the decontaminated signal vector $\hat{\mathbf{y}}_{k}[i]$ can be represented as:

$$
\begin{aligned}
\hat{\mathbf{y}}_{k}[i]= & \mathbf{R}^{T}[1] \mathbf{A}\left(\mathbf{b}[i+1]-\hat{\mathbf{b}}_{k}[i+1]\right) \\
& +\mathbf{R}[0]\left(\mathbf{b}[i]-\hat{\mathbf{b}}_{k}[i]\right) \\
& +\mathbf{R}[1]\left(\mathbf{b}[i-1]-\hat{\mathbf{b}}_{k}[i-1]\right) .
\end{aligned}
$$

According to Equation 13, we can observe that the decontaminated signal vector $\mathbf{y}[i]$ will be free from multiple alaccess interference, provided that the symbol estimates $\hat{\mathbf{b}}_{k}[i+1], \hat{\mathbf{b}}_{k}[i], \hat{\mathbf{b}}_{k}[i-1]$ are reliable. In comparison to the synchronous scenario of Equation 8 we can see that the iterative multiuser receiver communicating in an asynchronous scenario is more prone to error propagation than that of a synchronous scenario, because it requires three correctly estimated symbol vectors. Even if only one of the three vectors has errors, it will lead to increased residual interference. Hence the PIC based iterative multiuser receiver exhibits a slower convergence in the asynchronous environments than in the synchronous scenario.

\section{SIMULATION RESULTS}

For the sake of fair comparison, initially all the systems studied were configured for maintaining the same complexity. Specifically, a code memory of $v=6$ was invoked by the TCM scheme [6]. To ensure that the TTCM scheme exhibited a similar decoding complexity in comparison to the TCM scheme of memory $v=6$ expressed in terms of the number of decoding trellis states [6], a component TCM code memory of $v=3$ was used in the TTCM scheme. The number of decoding iterations was set to 4 for TTCM and there were 2 decoders, again, yielding a total of 64 trellis states. However, our initial investigations indicated that no significant performance improvements were achieved, when 4 TTCM iterations were invoked during all the PIC-based turbo iterations instead of 2 . Hence the complexity of the TTCM channel decoder was reduced by using only 2 iterations in all channel decoding processes apart from the last PIC-based turbo iteration of the iterative PIC-aided detector. The family of $m$-sequence spreading codes having a length of $N=15$ was employed in our system, and the transmission burst 
length was $T=120$ bits. A random channel interleaver having a memory of 8 transmission bursts was employed in our system. We will demonstrate that the system was capable of supporting $K=15$ users in a synchronous scenario, while attaining a single-user performance, which was reduced to $K=7$ users in an asynchronous context. Furthermore, we also investigated the performance of the PIC-based iterative receiver when communicating over an asynchronous two-path uncorrelated Rayleigh fading channel.

Figures 3 and 4 show the performance of the TTCM and TCM based 8PSK modulation scheme in both a synchronous and in an asynchronous scenario, respectively. We can observe that the iterative receiver is capable of approaching the single-user bound after two PIC-aided outer iterations, when communicating in a synchronous scenario, while four iterations are needed, when operating in an asynchronous context.

Furthermore, Figures 5 and 6 demonstrate that in a synchronous environment the PIC based iterative receiver requires three iterations for approaching the singleuser bound, when 16QAM is employed. By contrast, the number of the outer iterations has to be increased to four in an asynchronous scenario for sake of achieving a near single-user performance.

Finally, Figure 7 characterises the PIC based iterative receiver's performance, when communicating over an asynchronous two-path uncorrelated Rayleigh fading channel. As seen in Figure 7, the PIC based iterative receiver requires 5 iterations for approaching the single user performance at a BER of $10^{-4}$, while supporting users $K=10$.

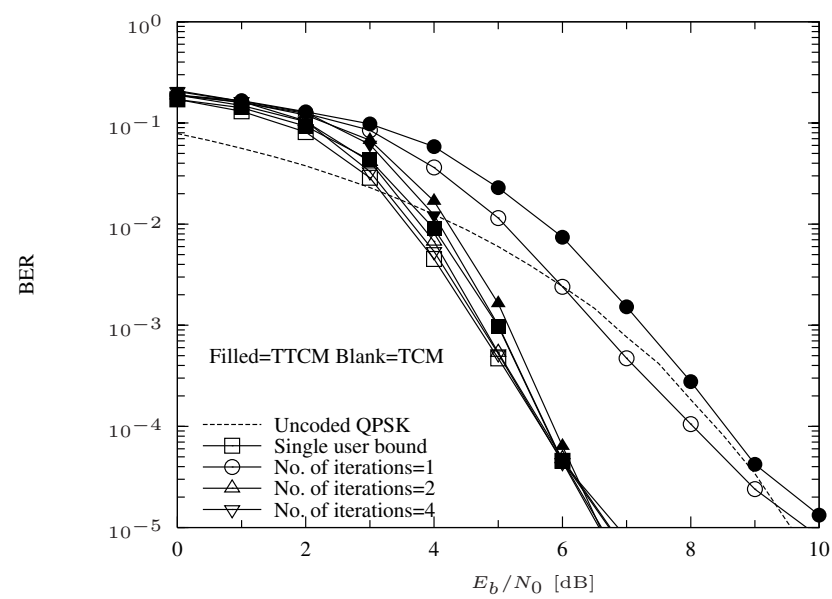

Figure 3: The PIC and TCM/TTCM based iterative multiuser detector's BER versus $E_{b} / N_{0}$ performance, when communicating over a synchronous non-dispersive AWGN channel, while supporting $K=15$ users. A $\frac{2}{3}$-rate 8PSK scheme having an effective throughput of 2 BPS was invoked.

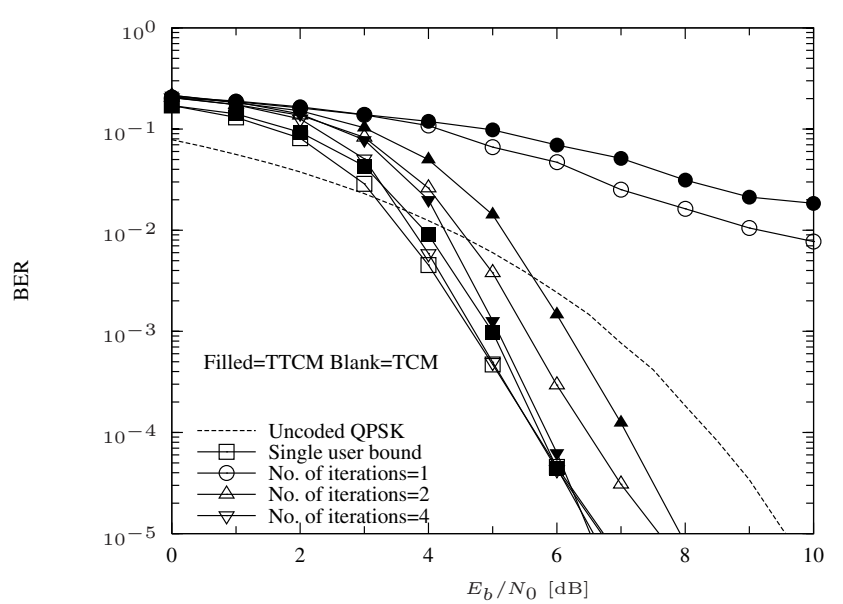

Figure 4: The PIC and TCM/TTCM based iterative multiuser detector's BER versus $E_{b} / N_{0}$ performance, when communicating over a asynchronous non-dispersive AWGN channel, while supporting $K=7$ users. A $\frac{2}{3}$ rate 8PSK scheme having an effective throughput of 2 BPS was invoked.

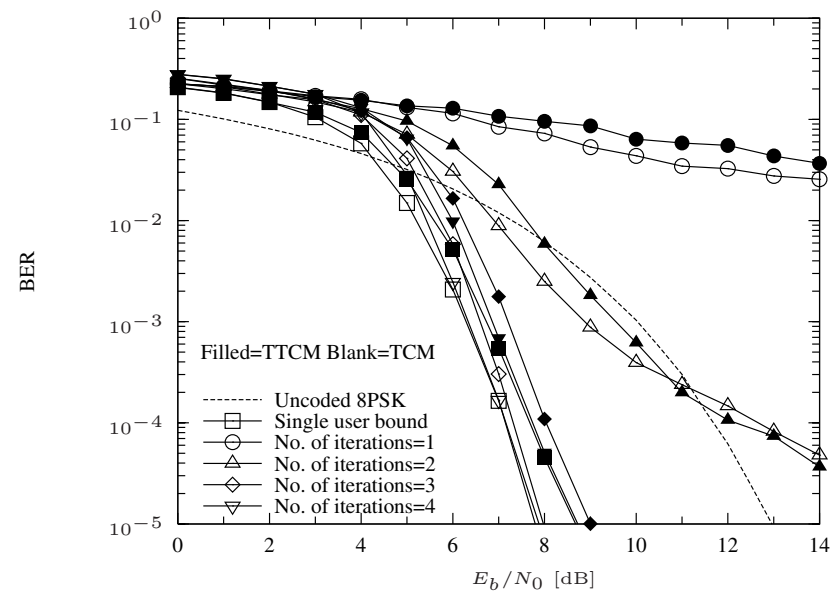

Figure 5: The PIC and TCM/TTCM based iterative multiuser detector's BER versus $E_{b} / N_{0}$ performance, when communicating over a synchronous non-dispersive AWGN channel, while supporting $K=15$ users. A $\frac{3}{4}$-rate 16QAM scheme having an effective throughput of 3 BPS was invoked. 


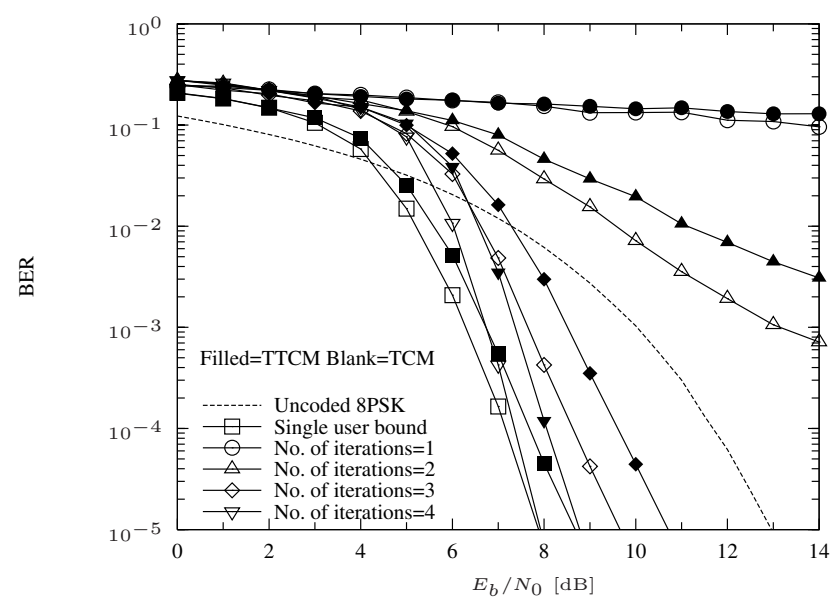

Figure 6: The PIC and TCM/TTCM based iterative multiuser detector's BER versus $E_{b} / N_{0}$ performance, when communicating over a asynchronous non-dispersive AWGN channel, while supporting $K=7$ users. A $\frac{3}{4}$ rate 16QAM scheme having an effective throughput of 3 BPS was invoked.

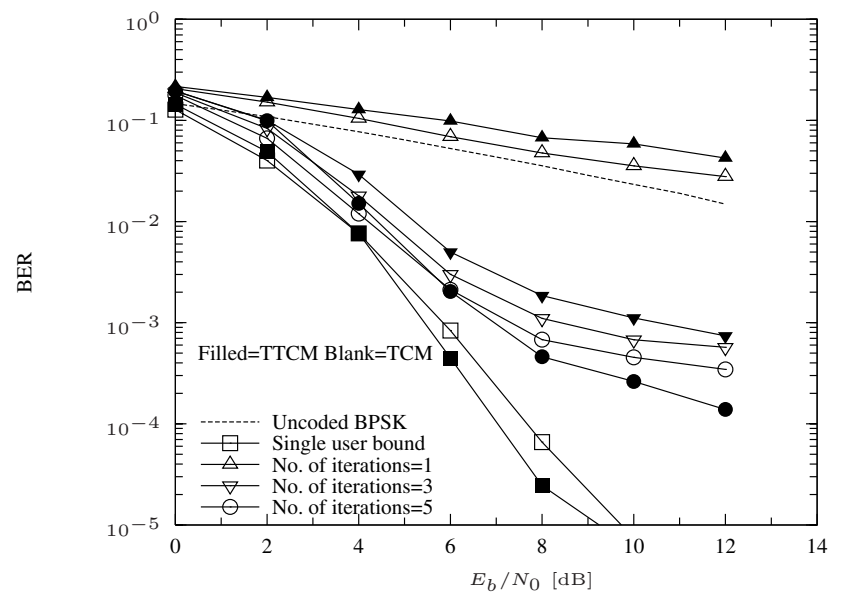

Figure 7: The PIC and TCM/TTCM based iterative multiuser detector's BER versus $E_{b} / N_{0}$ performance, when communicating over a asynchronous non-dispersive AWGN channel, while supporting $K=10$ users. A $\frac{1}{2}$ rate coded QPSK scheme having an effective throughput of 1 BPS was invoked.

\section{CONCLUSIONS}

The PIC based iterative multiuser detector's complexity per user bit is independent of the number of users supported, which is an attractive property. In the context of TCM and TTCM aided 8PSK modulation, the iterative receiver advocated is capable of approaching the singleuser performance bound after two PIC turbo iterations, when communicating in a synchronous AWGN scenario. By contrast, four PIC turbo iterations are necessitated, when communicating in an asynchronous AWGN channel. Furthermore, in the context of 16QAM the system approached the single-user bound after three iterations, when communicating in a synchronous environment, while requiring an increased number of four iterations when communicating in an asynchronous AWGN channel. Finally, the system requires 5 PIC turbo iterations, when transmitting over an asynchronous two-path Rayleigh fading channel.

\section{REFERENCES}

[1] C. Douillard, A. Picart, M. Jezeuel, P. Didier, C. Berrou, and A. Glavieux, "Iterative correction of intersymbol interference: Turbo-equalization," European Transactions on Telecommunications, vol. 6, pp. 507-511, SeptemberOctober 1995.

[2] M. C. Reed, C. B. Schlegel, P. D. Alexander, and J. A. Asenstorfer, "Iterative Multiuser Detection for CDMA with FEC: Near single user performance," IEEE Transactions on Communication, vol. 46, pp. 1693-1699, Dec. 1999.

[3] Y. Zhang, "Reduced complexity iterative multiuser detection for DS/CDMA with FEC," in International Conference on Universal Personal Communications, no. 12, (San Diego, U.S.A), pp. 10-14, Oct. 1997.

[4] P. D. Alexander, A. J. Grant, and M. C. Reed, "Performance analysis of an iterative decoder for code-division mulitple-access," European Transactions on Telecommunication, vol. 9, pp. 419-426, Sept./Oct 1998.

[5] M. C. Reed, Iterative Receiver Techniques for Coded Multiple Access Communication System. PhD thesis, The University of South Australia, 1999.

[6] L. Hanzo, T. H. Liew, and B. L. Yeap, Turbo Coding, Turbo Equalisation and Space-Time Coding for Transmission over Fading Channels. John Wiley-IEEE Press, 2002.

[7] A. Nordio, M. Hernandez, and G. Caire, "Design and performance of a low-complexity iterative multiuser joint decoder based on viterbi decoding and parallel interference cancellation," IEEE International Conference on Communications, vol. 6, pp. 298-302, 28 April-2 May 2002.

[8] J. Boutros and G. Caire, "Iterative multi-user joint decoding: Unified framework and asymptotic analysis," IEEE Transactions on Information Theory, vol. 48, pp. 17721793, July 2002.

[9] S. Verdu, Multiuser Detection. Cambridge Press, 1998. 